Internet Engineering Task Force (IETF)

J. Abley

ICANN

Request for Comments: 6305

Category: Informational

W. Maton

ISSN : 2070-1721

$\mathrm{NRC}-\mathrm{CNRC}$

July 2011

I'm Being Attacked by PRISONER. IANA. ORG!

Abstract

Many sites connected to the Internet make use of IPv4 addresses that are not globally unique. Examples are the addresses designated in RFC 1918 for private use within individual sites.

Hosts should never normally send DNS reverse-mapping queries for those addresses on the public Internet. However, such queries are frequently observed. Authoritative servers are deployed to provide authoritative answers to such queries as part of a loosely coordinated effort known as the AS112 project.

Since queries sent to AS112 servers are usually not intentional, the replies received back from those servers are typically unexpected. Unexpected inbound traffic can trigger alarms on intrusion detection systems and firewalls, and operators of such systems often mistakenly believe that they are being attacked.

This document provides background information and technical advice to those firewall operators.

Status of This Memo

This document is not an Internet Standards Track specification; it is published for informational purposes.

This document is a product of the Internet Engineering Task Force (IETF). It represents the consensus of the IETF community. It has received public review and has been approved for publication by the Internet Engineering Steering Group (IESG). Not all documents approved by the IESG are a candidate for any level of Internet Standard; see Section 2 of RFC 5741.

Information about the current status of this document, any errata, and how to provide feedback on it may be obtained at http://www.rfc-editor.org/info/rfc6305. 
Copyright Notice

Copyright (c) 2011 IETF Trust and the persons identified as the document authors. All rights reserved.

This document is subject to BCP 78 and the IETF Trust's Legal Provisions Relating to IETF Documents (http://trustee.ietf.org/license-info) in effect on the date of publication of this document. Please review these documents carefully, as they describe your rights and restrictions with respect to this document. Code Components extracted from this document must include Simplified BSD License text as described in section $4 . e$ of the Trust Legal Provisions and are provided without warranty as described in the Simplified BSD License.

Table of Contents

1. Introduction and Target Audience . . . . . . . . . . . . . 3

2. Private-Use Addresses . . . . . . . . . . . . . . . . . . 3

3. DNS Reverse Mapping . . . . . . . . . . . . . . . . . . . 3

4. DNS Reverse Mapping for Private-Use Addresses . . . . . . . . . 4

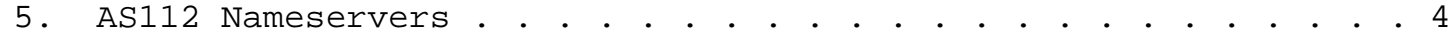

6. Inbound Traffic from AS112 Servers . . . . . . . . . . . . . 5

7. Corrective Measures . . . . . . . . . . . . . . . . 5

8. AS112 Contact Information . • • . . . . . . . . . . . . . 6

9. IANA Considerations . . . . • . . . . . . . . . . . . . . . 6

10. Security Considerations . . . . . . . . . . . . . . . . 7

11. Acknowledgements . . . . . . . . . . . . . . . . . . 7

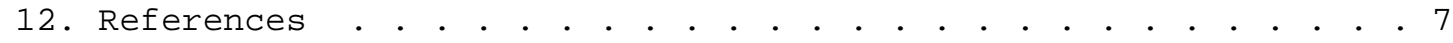
12.1. Normative References . • • • • • . . . . . . . . . . . 7

12.2. Informative References . . . . . . . . . . . . . . . 7 


\section{Introduction and Target Audience}

Readers of this document may well have experienced an alarm from a firewall or an intrusion-detection system, triggered by unexpected inbound traffic from the Internet. The traffic probably appeared to originate from one of several hosts discussed further below.

The published contacts for those hosts may well have suggested that you consult this document.

If you are following up on such an event, you are encouraged to follow your normal security procedures and take whatever action you consider to be appropriate. This document contains information that may assist you.

2. Private-Use Addresses

Many sites connected to the Internet make use of address blocks designated in [RFC1918] for private use. One example of such addresses is 10.1 .30 .20 .

Because these ranges of addresses are used by many sites all over the world, each individual address can only ever have local significance. For example, the host numbered 192.168.18.234 in one site almost certainly has nothing to do with a host with the same address located in a different site.

\section{DNS Reverse Mapping}

The Domain Name System (DNS) [RFC1034] can be used to obtain a name for a particular network address. The process by which this happens is as follows:

1. The network address is rearranged in order to construct a name that can be looked up in the DNS. For example, the IPv4 address 10.1.30.20 corresponds to the DNS name 20.30.1.10.IN-ADDR.ARPA.

2. A DNS query is constructed for that name, requesting a DNS record of the type "PTR".

3. The DNS query is sent to a resolver.

4. If a response is received in response to the query, the answer will typically indicate either the hostname corresponding to the network address, or the fact that no hostname can be found.

This procedure is generally carried out automatically by software, and hence is largely hidden from users and administrators. 
Applications might have reason to look up an IP address in order to gather extra information for a log file, for example.

4. DNS Reverse Mapping for Private-Use Addresses

As noted in Section 2, private-use addresses have only local significance. This means that sending queries out to the Internet is not sensible: there is no way for the public DNS to provide a useful answer to a question that has no global meaning.

Despite the fact that the public DNS cannot provide answers, many sites have misconfigurations in the way they connect to the Internet; this results in such queries relating to internal infrastructure being sent outside the site. From the perspective of the public DNS, these queries are junk -- they cannot be answered usefully and result in unnecessary traffic being received by the nameservers which underpin the operation of the reverse DNS (the so-called reverse servers [RFC5855], which serve "IN-ADDR.ARPA").

To isolate this traffic and reduce the load on the rest of the reverse DNS infrastructure, dedicated servers have been deployed in the Internet to receive and reply to these junk queries. These servers are deployed in many places in a loosely coordinated effort known as the "AS112 project". More details about the AS112 project can be found at <http://www.as112.net/>.

5. AS112 Nameservers

The nameservers responsible for answering queries relating to private-use addresses are as follows:

O PRISONER. IANA.ORG (192.175.48.1)

O BLACKHOLE-1.IANA.ORG (192.175.48.6)

○ BLACKHOLE-2. IANA.ORG (192.175.48.42)

A request sent to one of these servers will result in a response being returned to the client. The response will typically be a UDP datagram, although it's perfectly valid for requests to be made over TCP. In both cases, the source port of packets returning to the site that originated the DNS request will be 53 . 


\section{Inbound Traffic from AS112 Servers}

Where firewalls or intrusion detection systems (IDSs) are configured to block traffic received from AS112 servers, superficial review of the traffic may seem alarming to site administrators.

- Since requests directed ultimately to AS112 servers are usually triggered automatically by applications, review of firewall logs may indicate a large number of policy violations occurring over an extended period of time.

- Where responses from AS112 servers are blocked by firewalls, hosts will often retry, often with a relatively high frequency. This can cause inbound traffic to be misclassified as a denial-ofservice (DoS) attack. In some cases, the source ports used by individual hosts for successive retries increase in a predictable fashion (e.g. monotonically), which can cause the replies from the AS112 server to resemble a port scan.

- A site administrator may attempt to perform active measurement of the remote host in response to alarms raised by inbound traffic, e.g. initiating a port scan in order to gather information about the host which is apparently attacking the site. Such a scan will usually result in additional inbound traffic to the site performing the measurement, e.g., an apparent flood of ICMP messages that may trigger additional firewall alarms and obfuscate the process of identifying the originally problematic traffic.

\section{Corrective Measures}

A site that receives responses from one of the nameservers listed in Section 5 is probably under no immediate danger, and the traffic associated with those responses probably requires no emergency action by the site concerned. However, this document cannot aspire to dictate the security policy of individual sites, and it is recognised that many sites will have perfectly valid policies that dictate that corrective measures should be taken to stop the responses from AS112 servers.

It should be noted, however, that the operators of AS112 nameservers, which are generating the responses described in this document, are not ultimately responsible for the inbound traffic received by the site: that traffic is generated in response to queries that are sent out from the site, and so the only effective measures to stop the inbound traffic is to prevent the original queries from being made. 
Possible measures that might be taken to prevent these queries include:

1. Stop hosts from making these DNS reverse-mapping queries in the first place. In some cases, servers can be configured not to perform DNS reverse-mapping lookups, for example. As a general site-wide approach, however, this measure is frequently difficult to implement due to the large number of hosts and applications involved.

2. Block DNS reverse-mapping queries to the AS112 servers from leaving the site using firewalls between the site and the Internet. Although this might appear to be sensible, such a measure might have unintended consequences: the inability to receive an answer to DNS reverse-mapping queries might lead to long DNS lookup timeouts, for example, which could cause applications to malfunction. (It may also lead to the belief that the Internet or the local network is down.)

3. Configure all DNS resolvers in the site to answer authoritatively for the zones corresponding to the private-use address blocks in use. This should prevent resolvers from ever needing to send these queries to the public DNS. Guidance and recommendations for this aspect of resolver configuration can be found in [RFC6303].

4. Implement a private AS112 node within the site. Guidance for constructing an AS112 node may be found in [RFC6304].

8. AS112 Contact Information

More information about the AS112 project can be found at <http://www.as112.net/>.

\section{IANA Considerations}

The AS112 nameservers are all named under the domain IANA.ORG (see Section 5). The IANA is the organisation responsible for the coordination of many technical aspects of the Internet's basic infrastructure. The AS112 project nameservers provide a public service to the Internet that is sanctioned by and operated in loose coordination with the IANA. 


\section{Security Considerations}

The purpose of this document is to help site administrators properly identify traffic received from AS112 nodes and to provide background information to allow appropriate measures to be taken in response to it.

Hosts should never normally send queries to AS112 servers: queries relating to private-use addresses should be answered locally within a site. Hosts that send queries to AS112 servers may well leak information relating to private infrastructure to the public network; this could represent a security risk.

\section{Acknowledgements}

The authors wish to acknowledge the assistance of $\mathrm{S}$. Moonesamy in the preparation of this document.

12. References

12.1. Normative References

[RFC1034] Mockapetris, P., "Domain names - concepts and facilities", STD 13, RFC 1034, November 1987.

[RFC1918] Rekhter, Y., Moskowitz, R., Karrenberg, D., Groot, G., and E. Lear, "Address Allocation for Private Internets", BCP 5, RFC 1918, February 1996.

12.2. Informative References

[RFC5855] Abley, J. and T. Manderson, "Nameservers for IPv4 and IPv6 Reverse Zones", BCP 155, RFC 5855, May 2010.

[RFC6303] Andrews, M., "Locally Served DNS Zones", BCP 163, REC 6303, July 2011.

[RFC6304] Abley, J. and W. Maton, "AS112 Nameserver Operations", RFC 6304, July 2011. 


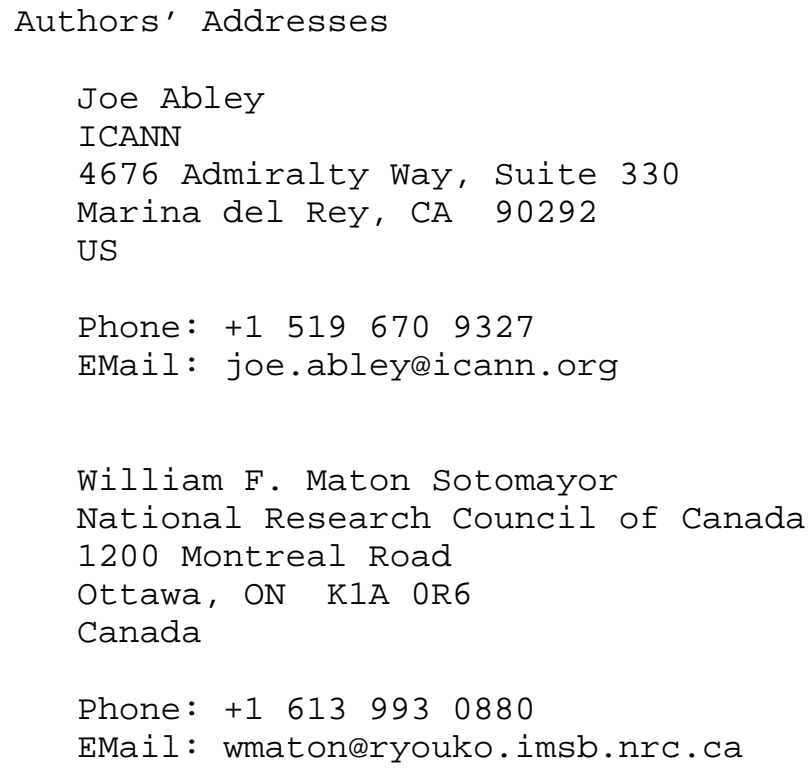

